\title{
Observation of Adsorption and Desorption of Water Molecules with Ambient SIMS
}

Jiro Matsuo ${ }^{1,2}$

1. Quantum Science and Engineering Center, Kyoto University, Uji, Kyoto, Japan.

2. SENTAN, Japan Science and Technology Agency (JST), Tokyo, Japan.

Water is the most important liquid on earth. It circulates in the earth and is used by living organisms. Water has a rather high vapor pressure $(>1000 \mathrm{~Pa})$, and phenomena of absorption and desorption of water molecules on and from solid surfaces are often observed in our daily life. The physical and chemical properties of the water molecule, its surface in liquid state and at water interfaces with solids are interesting fields of investigation. However, the use of conventional surface and interface analytical techniques that require high vacuum conditions is not possible with water, because of its high vapor pressure. Moreover, most liquids are organic materials, for which chemical analysis is more important than elemental analysis. Thus, a new analytical technique for water that works in ambient conditions is strongly required.

Secondary ion mass spectrometry (SIMS) is one of the analytical techniques for surfaces and interfaces that is widely used for analysis of materials, such as polymers and biological samples, as well as semiconductors and ceramics. The low energy $(\sim \mathrm{keV})$ ion beams that are used in conventional SIMS systems are difficult to transport in the ambient. We have developed a SIMS technique that uses swift heavy ion beams (MeV-SIMS) that have a high transmission capability in matter and can be extracted under ambient conditions. In the conventional SIMS technique, the use of low-energy (keV) ion excitation of nuclei is dominant, but in the high energy range (high-energy, $>\mathrm{MeV}$ ), most of the energy deposited by the incoming ions is used for electronic excitation. Because of the different excitation mechanism in energy deposition, secondary molecular ion emission is significantly enhanced with swift heavy ion beams [1]. This is quite essential for analyzing structures of organic molecules.

We have demonstrated the technique of molecular imaging with swift heavy ion beams (MeV-SIMS) in biological material analysis. The molecular distribution (up to $1 \mathrm{kDa}$ ) was clearly imaged with a lateral resolution of around $5 \mu \mathrm{m}$, opening new opportunities for chemical imaging. The MeV-SIMS technique is now being developed in various ion accelerator laboratories in the world and is being established as a new ion beam analysis (IBA) technique.

A specially designed SIMS system was constructed at Kyoto University for ambient SIMS measurements [2]. In this apparatus, a $6-\mathrm{MeV} \mathrm{Cu}$ ion beam is introduced into a target chamber that is kept at ambient conditions. The secondary molecular ions emitted from the sample surface are extracted through a small orifice into a differentially pumped chamber and measured with an orthogonal acceleration time-of-flight (oa-TOF) mass spectrometer (Fig. 1). The target chamber is kept at ambient conditions $(100,000 \mathrm{~Pa})$ with varying humidity levels $(0-100 \%)$. One of the key technologies is maintaining the vacuum pressure in the TOF chamber. A difference of more than ten orders of magnitude in vacuum pressure was achieved. The suppression of chemical noise in the spectra is another issue. This noise originates from various molecules in the ambient, because these molecules acquire energy from the $\mathrm{MeV}$ ions and become ionized. The electric field is manipulated to suppress extraction of such ions by adjusting the voltage of the sample and the extraction cone. 
Ambient SIMS spectra of a Si wafer obtained with swift heavy ions under wet and dry conditions are shown in Fig. 2. In the wet condition, water clusters were found, indicating a liquid water layer. This spectrum is quite similar to that of liquid water (droplets). In contrast, the total ion intensity was rather low and the highest peak was found at $\mathrm{m} / \mathrm{z}=32$, which is oxygen molecules in the ambient. The $\left[\mathrm{H}_{2} \mathrm{O}+\mathrm{H}\right]^{+}$peak was also found in the spectrum, but the multimer of water molecules was very weak. These results were quite consistent with results of chemisorption of one monolayer of water molecules on a Si surface in high-vacuum condition. In contrast, more than one monolayer of water was adsorbed on the Si surface in the wet ambient condition.

The new system allows measurements of adsorption and desorption of volatile molecules, liquid surfaces and liquid-solid interfaces in the ambient. However, the emission mechanism of large intact molecular ions is still to be clarified and the interpretation of SIMS spectra from liquids is not yet established.

Recent progress on this technique will be presented and discussed along with possible applications for liquid analysis.

References:

[1] J. Matsuo et al, Surf. Interface Anal. 42 (2010), p. 1612.

[2] M. Kusakari et al, J. Vac. Sci and Tech. B 34 (2016), p. 034H111.

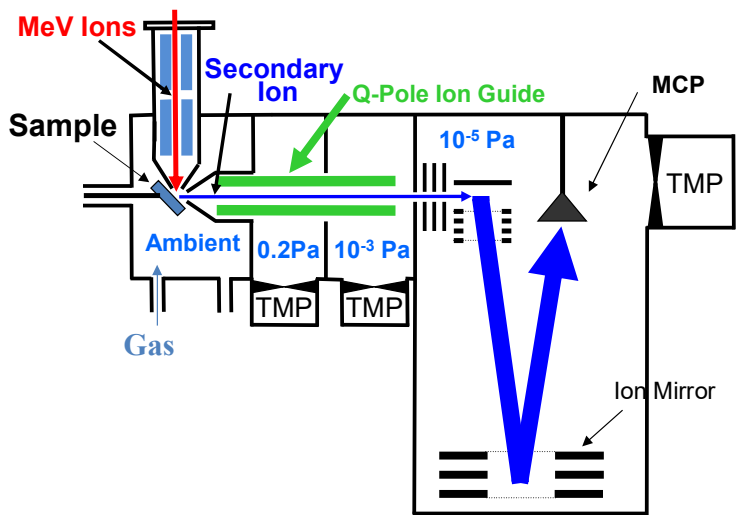

Figure 1. Schematic diagram of the Ambient-SIMS System with oa-TOF.

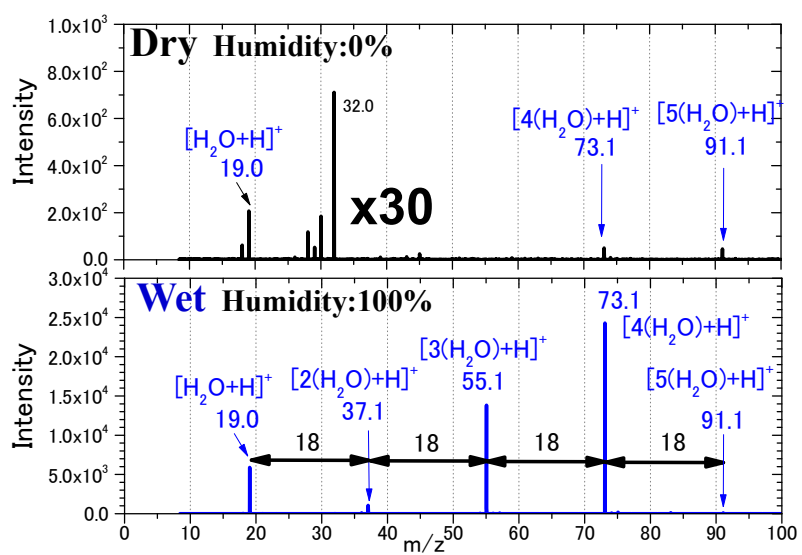

Figure 2. SIMS spectra under dry and wet condition; water-related peaks were clearly observed under wet condition. 